\title{
Impact of antimicrobial therapy on prognosis of patients requiring valve surgery during active infective endocarditis
}

\author{
Georges Fayad, MD, ${ }^{a}$ Andre Vincentelli, MD, PhD, ${ }^{a}$ Guillaume Leroy, MD, ${ }^{b}$ Patrick Devos, MD, ${ }^{c}$ \\ Gilles Amr, MD, ${ }^{\mathrm{a}}$ Alain Prat, MD, ${ }^{\mathrm{a}}$ Mohammad Koussa, MD, ${ }^{\mathrm{a}}$ and Olivier Leroy, $\mathrm{MD}^{\mathrm{b}}$
}

Objectives: We examined the characteristics and outcomes of patients requiring valve surgery during active infective endocarditis (IE), focusing on the impact of antimicrobial therapy.

\begin{abstract}
Methods: In this retrospective study, medical charts of all consecutive patients admitted to our cardiovascular surgery department from January 1998 to December 2010, with a diagnosis of IE requiring surgical management, were reviewed. Adult patients were enrolled in the study if they had definite or possible active IE and if the antimicrobial treatment was evaluable.

Results: After initial screening of medical records, we selected 173 surgically treated patients (135 men; mean age, 55.8 years). Native valves were involved in $150(87 \%)$ patients. IE mainly involved the aortic valve $(n=113)$ and then mitral $(n=83)$, tricuspid $(n=13)$, and pulmonary $(n=3)$ valves. The most common causative pathogens were streptococci $(n=70)$, staphylococci $(n=60)$, and enterococci $(n=29)$. Operative mortality was $15 \%$. Multivariate logistic regression analysis demonstrated that adequacy of the overall antimicrobial treatment (adjusted odds ratio, $0.292 ; 95 \%$ confidence interval, $0.117-0.726 ; P=.008)$ and temperature greater than $38^{\circ} \mathrm{C}$ at the time of diagnosis (adjusted odds ratio, $0.288 ; 95 \%$ confidence interval, $0.115-0.724 ; P=.008$ ) were independently associated with a lower risk of mortality. Conversely, age greater than 60 years (adjusted odds ratio, 4.42; $95 \%$ confidence interval, $1.57-12.4 ; P=.005$ ) was associated with a greater risk of operative mortality.
\end{abstract}

Conclusions: Surgery for active IE is still associated with a high mortality rate, but its prognosis is significantly improved by adequate antimicrobial therapy. (J Thorac Cardiovasc Surg 2014;147:254-8)

The optimal management of infective endocarditis (IE) requires a collaborative approach, notably involving specialists in microbiology, infectious diseases, cardiology, cardiac surgery, and anaesthesiology.

Even if antimicrobial treatment represents the cornerstone of management, recent data from the International Collaboration on Endocarditis-Prospective Cohort Study ${ }^{1}$ have shown that $1(48 \%)$ of 2 patients required valve surgery. Perioperative mortality varies between $5 \%$ and $36 \%{ }^{2-10}$ Prognosis depends on the causative organism, the extent of destruction of cardiac structures, the presence of complications of IE such as heart failure, and the timing of surgery. ${ }^{11}$

Several studies have demonstrated that adequate antibiotic therapy improves the outcome of patients with severe bacterial or fungal infection and that the involvement of an infectious disease specialist could potentially increase adequate antibiotic use. ${ }^{12-15}$

\footnotetext{
From Centre Hospitalier Régional Universitaire de Lille, ${ }^{a}$ Pôle de Chirurgie Cardiovasculaire, Hôpital Cardiologique, Lille; Service de Réanimation et Maladies Infectieuses, ${ }^{\mathrm{b}}$ Centre Hospitalier Chatiliez, Tourcoing; and Département de BioStatistiques, ${ }^{\mathrm{c}} \mathrm{CHRU}$, Lille, France.

Disclosures: Authors have nothing to disclose with regard to commercial support.

Received for publication Aug 13, 2012; revisions received Sept 14, 2012; accepted for publication Oct 10, 2012; available ahead of print Nov 9, 2012.

Address for reprints: Georges Fayad, MD, Pôle de Chirurgie Cardiovasculaire, Hôpital Cardiologique, CHRU de Lille, France (E-mail: g.fayad@sfr.fr).

$0022-5223 / \$ 36.00$

Copyright (c) 2014 by The American Association for Thoracic Surgery

http://dx.doi.org/10.1016/j.jtcvs.2012.10.019
}

The goal of the present study was to identify predictive factors of mortality while focusing on the impact of antimicrobial therapy in a cohort of consecutive patients operated on through a 13-year experience for active IE.

\section{PATIENTS AND METHODS} Study Design and Patients

A systematic retrospective review was carried out of all consecutive patients admitted to the 2 units of the Cardiovascular Surgery Department of Hôpital Cardiologique de Lille, France, from January 1998 to December 2010, with a diagnosis of IE requiring surgical management. All medical charts were analyzed by an independent group (Centre d'Investigation Clinique, CHRU, Lille, France). In accordance with French law, no ethics committee approval was required to conduct the study.

Adult patients were enrolled in the study if they had definite or possible active IE and if the antimicrobial treatment of the episode of IE was evaluable. Definite or possible IE was defined according to modified Duke criteria. ${ }^{16}$ Endocarditis was defined as active if the patient required surgery before completion of a standard course of antibiotic treatment, irrespective of whether there were ongoing signs of sepsis or whether blood and valve cultures were positive for the infecting microorganism. ${ }^{2}$ Antimicrobial treatment of the episode of IE was considered evaluable when the causative organism(s), antibiotics used, and duration of postoperative antimicrobial treatment were clearly identified. Patients with IE associated with pacemaker implantation were excluded.

\section{Data Collection and Definitions}

Data were recorded on a standardized report form designed by 2 of the authors (G.F., O.L.). Information about patient demographics, preexisting conditions, focus of infection, initial valve status, clinical course, echocardiographic data, microbiologic data, complications, cardiac surgery 


\section{Abbreviations and Acronyms \\ ICU $=$ intensive care unit \\ IE = infective endocarditis}

interventions, antimicrobial therapy during the preoperative and the postoperative periods, and outcome were collected.

Prosthetic valve IE was defined as infection occurring on any type of tissue or mechanical device. Hospital-acquired IE was defined as infection occurring more than 72 hours after admission to the hospital or acquired in association with a significant invasive procedure performed during a recent hospitalization within 8 weeks of this hospitalization. ${ }^{17}$ Antimicrobial therapy was considered as bacteriologically effective if it included antibiotic(s) usually proposed by current guidelines (ie, amoxicillin or a thirdgeneration cephalosporin or a glycopeptide in the case of IE owing to Streptococcus spp; amoxicillin or a glycopeptide for IE owing to Enterococcus spp; oxacillin for IE owing to methicillin-susceptible Staphylococcus spp; a glycopeptide for IE owing to methicillin-resistant Staphylococcus spp; and a third-generation cephalosporin or fluoroquinolone for IE owing to gram-negative bacilli) and if the causative organism was in vitro susceptible to at least 1 of the antibiotics of the regimen. ${ }^{18}$ The duration of postoperative antimicrobial treatment was considered correct when it was 2 weeks or more when the leaflet culture was negative and 6 weeks when leaflet culture was positive. The antimicrobial treatment was considered adequate when it was bacteriologically effective in both the preoperative and postoperative periods and had a correct postoperative duration. Operative mortality was defined as death occurring within the same hospitalization as surgery, regardless of its cause. . $^{2,3}$

\section{Statistical Analysis}

Descriptive analyses were performed to check and summarize the data. Quantitative variables are reported as means \pm standard deviation. Qualitative variables are reported as number and percentage. Continuous variables were compared using the Student $t$ test. Categorical variables were compared using the $\chi^{2}$ test or Fisher's exact test when $\chi^{2}$ was not appropriate.

A stepwise logistic regression analysis was performed including variables with a $P$ value $\leq .10$ in univariate analysis. Adjusted odds ratios were computed using logistic regression analysis including the independent predictors of mortality.

All statistical analyses were performed using SAS software, version 9.1 (SAS Institute, Inc, Cary, NC).

\section{RESULTS}

After initial screening of 371 medical records, we excluded 140 patients who either had not had active IE or did not meet modified Duke criteria for diagnosis, and we retained 231 patients exhibiting definite or possible active IE. Among them, the antimicrobial treatment of the episode of IE was evaluable in 173 patients who met the eligibility criteria for inclusion in this study.

Most patients had definite active IE $(\mathrm{n}=169 ; 98 \%)$. Blood and leaflet cultures were positive in $168(97 \%)$ and $23(13 \%)$ cases, respectively. Echocardiography demonstrated endocardial involvement in $163(94 \%)$ cases. Duke minor criteria observed were predisposition $(\mathrm{n}=57)$, fever $(\mathrm{n}=127)$, and vascular $(\mathrm{n}=18)$ and immunologic phenomena $(\mathrm{n}=7)$. IE was hospital acquired in 16 $(9 \%)$ cases. Demographics characteristics, underlying valve diseases, and portal of entry are reported in Table 1. Most patients $(\mathrm{n}=130 ; 75 \%)$ were referred from another hospital.

Native and prosthetic valves were involved in $150(87 \%)$ and $23(13 \%)$ patients, respectively. IE mainly involved the aortic valve $(\mathrm{n}=113)$ and then mitral $(\mathrm{n}=83)$, tricuspid $(\mathrm{n}=13)$, and pulmonary $(\mathrm{n}=3)$ valves. Multiple valve involvement was observed in $38(22 \%)$ patients. Valves involved were aortic plus mitral valves $(\mathrm{n}=26)$, aortic plus tricuspid valves $(\mathrm{n}=3)$, aortic plus pulmonary valves $(\mathrm{n}=3)$, mitral plus tricuspid valves $(\mathrm{n}=5)$, and aortic plus mitral plus tricuspid valves $(\mathrm{n}=1)$.

One hundred eighty-eight causative pathogens were identified. IE was polymicrobial in 13 patients. The most common were streptococci $(\mathrm{n}=70 ; 37 \%)$, staphylococci $(n=60 ; 31.5 \%)$, and enterococci $(n=29 ; 15.5 \%)($ Table 2$)$.

During the course of IE and before the surgical procedure, $84(49 \%)$ patients exhibited extracardiac involvement. Main organs involved were central nervous system $(\mathrm{n}=49)$, spleen $(\mathrm{n}=34)$, lung $(\mathrm{n}=13)$, bone and joints $(\mathrm{n}=13)$, kidneys $(\mathrm{n}=10)$, and liver $(\mathrm{n}=3)$.

Surgery was performed in the acute phase of IE with a mean delay from diagnosis to surgery of $22.9 \pm 17.5$ days. Main indications for surgery were 1 or more of the following reasons: persistent infection despite 7 to 10 days of bacteriologically effective antibiotic treatment $(n=100)$, vegetation size $(\mathrm{n}=50)$, congestive heart failure $(\mathrm{n}=60)$, recurrent embolization $(\mathrm{n}=22)$, annular abscess $(\mathrm{n}=21)$, and conduction abnormalities $(\mathrm{n}=5)$. A total of 86 bioprostheses, 63 mechanical prostheses, and 9 aortic homografts were implanted. After excision of the vegetations, valve repair was performed alone $(\mathrm{n}=15)$ or in association with prosthesis implantation $(\mathrm{n}=7)$ in 22 cases.

After the surgical procedure, all patients were admitted to the surgical intensive care unit (ICU). Vasopressors and/or inotropes were required in $99(57 \%)$ cases. Renal replacement therapy was required in 19 cases. The mean duration of postoperative mechanical ventilation was $55.8 \pm 96.4$ hours. The mean duration of ICU stay was $150 \pm 152$ hours.

All patients received a preoperative antimicrobial treatment. Its mean duration before surgery was $21.4 \pm 14.5$ days. This antimicrobial treatment was bacteriologically effective in $159(92 \%)$ patients. In 7 patients, antibiotics used were not usually proposed by current guidelines. In the remaining 7 patients, at least 1 causative pathogen was resistant to all antibiotics of the regimen. In 1 case, this point was explained by a late postoperative identification of causative pathogen. Blood cultures were negative whereas leaflet culture identified a pathogen resistant to the preoperative antibiotic regimen. Postoperative antimicrobial treatment was bacteriologically effective in $163(94 \%)$ cases. In 10 patients, either antibiotics used were not usually proposed by current guidelines $(n=5)$ or at least 1 causative pathogen was resistant to all antibiotics of the regimen $(n=5)$. 
TABLE 1. Demographic characteristics of the study population $(\mathbf{n}=\mathbf{1 7 3})$

\begin{tabular}{lc}
\hline \multicolumn{1}{c}{ Characteristic } & n $(\%)$ or mean $( \pm$ SD $)$ \\
\hline Men & $135(78)$ \\
Age $(y)$ & $55.8 \pm 15.9$ \\
Underlying valve diseases & $53(31)$ \\
$\quad$ Mitral insufficiency & 9 \\
Aortic insufficiency & 12 \\
Aortic stenosis & 7 \\
Valve prosthesis & 23 \\
Other & 2 \\
History of IE & $4(2)$ \\
Identified portal of entry & $87(50)$ \\
Dental or upper respiratory tract & $40(23)$ \\
Cutaneous & $17(10)$ \\
Digestive tract & $14(8)$ \\
Intravenous drug use & $4(2)$ \\
Cardiovascular procedure or vascular access & $10(6)$ \\
Genitourinary tract & $4(2)$ \\
\hline$S D$, Standard deviation; $I E$, infective endocarditis. &
\end{tabular}

The duration of postoperative antimicrobial treatment was correct in $135(78 \%)$ cases. In 38 cases, treatment was stopped too early. Finally, the overall antimicrobial treatment was considered adequate in $122(70.5 \%)$ patients.

During hospitalization, 26 (15\%) patients died. Bivariate analysis of main risk factors for operative mortality is reported in Table 3. Nonsurvivors were significantly older $(65.7 \pm 9.4$ vs $54.0 \pm 16.3$ years $)$ and the mortality rate was significantly higher when temperature was less than $38^{\circ} \mathrm{C}$ at the time of diagnosis ( $28.3 \%$ vs $\left.10.2 \%\right)$, IE involved a prosthetic valve $(30.5 \%$ vs $12.7 \%)$, preoperative antimicrobial treatment was not bacteriologically effective $(50 \%$ vs $12 \%$ ), duration of postoperative antimicrobial treatment was not correct $(26.3 \%$ vs $11.8 \%)$, and overall antimicrobial treatment was not adequate $(27.5 \%$ vs $9.8 \%)$.

The results of multivariate analysis are reported in Table 4. Age older than 60 years, temperature greater than $38^{\circ} \mathrm{C}$ at the time of diagnosis, and adequacy of the overall antimicrobial

TABLE 2. Causative microorganisms $(n=188)$ isolated from cases of active IE

\begin{tabular}{lc}
\hline \multicolumn{1}{c}{ Micro organism } & $\mathbf{n}(\%)$ \\
\hline Streptococcus spp & $70(37)$ \\
Enterococcus spp & $29(15.5)$ \\
Staphylococcus aureus & $40(21)$ \\
$\quad$ Methicillin-susceptible & 35 \\
Methicillin-resistant & 5 \\
Coagulase-negative Staphylococcus & $20(10.5)$ \\
Gram-negative bacilli & $20(10.5)$ \\
Haemophilus influenzae & 6 \\
Other & 14 \\
Gram-positive bacilli & $5(2.5)$ \\
Candida spp & $3(1.5)$ \\
Aspergillus spp & $1(0.5)$ \\
\hline IE Infective
\end{tabular}

IE, Infective endocarditis; $s p p$, species.
TABLE 3. Bivariate analysis of risk factors for operative mortality

\begin{tabular}{|c|c|c|c|}
\hline Factor & $\begin{array}{l}\text { Survivors } \\
(\mathrm{n}=147)\end{array}$ & $\begin{array}{c}\text { Nonsurvivors } \\
(\mathbf{n}=\mathbf{2 6})\end{array}$ & $\begin{array}{c}P \\
\text { value }\end{array}$ \\
\hline Male gender & 117 & 18 & .3 \\
\hline Mean age (y) & $54.0 \pm 16.3$ & $65.7 \pm 9.4$ & $<.0001$ \\
\hline Age $>60 y$ & 67 & 20 & .003 \\
\hline $\begin{array}{l}\text { Temperature }>38^{\circ} \mathrm{C} \text { at the time } \\
\text { of diagnosis }\end{array}$ & 114 & 13 & .006 \\
\hline Aortic IE & 98 & 15 & .38 \\
\hline Mitral IE & 69 & 14 & .53 \\
\hline Multiple valve IE & 34 & 4 & .53 \\
\hline Prosthetic valve endocarditis & 16 & 7 & .05 \\
\hline Staphylococcal IE & 48 & 9 & .84 \\
\hline Streptococcal IE & 86 & 13 & .42 \\
\hline Gram-negative bacilli IE & 15 & 3 & .74 \\
\hline Fungal IE & 2 & 2 & .1 \\
\hline Polymicrobial IE & 11 & 2 & 1 \\
\hline Hospital-acquired IE & 14 & 2 & 1 \\
\hline Periannular complications & 28 & 7 & .36 \\
\hline Vegetations $>15 \mathrm{~mm}$ & 39 & 5 & .43 \\
\hline Neurologic complications & 41 & 8 & .76 \\
\hline Extracardiac involvement & 72 & 12 & .95 \\
\hline $\begin{array}{l}\text { Preoperative antimicrobial } \\
\text { treatment bacteriologically } \\
\text { effective }\end{array}$ & 140 & 19 & .001 \\
\hline $\begin{array}{l}\text { Postoperative antimicrobial } \\
\text { treatment bacteriologically } \\
\text { effective }\end{array}$ & 139 & 24 & .65 \\
\hline $\begin{array}{l}\text { Correct duration of postoperative } \\
\text { antimicrobial treatment }\end{array}$ & 119 & 16 & .04 \\
\hline $\begin{array}{l}\text { Adequacy of the overall } \\
\text { antimicrobial treatment }\end{array}$ & 110 & 12 & .003 \\
\hline
\end{tabular}

treatment are the independent predictors of operative mortality. The first is associated with an increased mortality, whereas the others are associated with an increased survival.

\section{DISCUSSION}

This study underlines the high mortality rate associated with surgery during the active phase of IE and demonstrates that an adequate antimicrobial treatment associated with surgery is an independent factor improving the outcome.

The recent European guideline on the prevention, diagnosis, and treatment of IE emphasizes the high in-hospital

TABLE 4. Multivariate analysis for independent predictors for operative mortality

\begin{tabular}{lccc}
\hline \multicolumn{1}{c}{ Parameter } & AOR & $\mathbf{9 5} \%$ CI & $\boldsymbol{P}$ value \\
\hline Age $>60$ y & 3.71 & $1.35-10.20$ & .01 \\
$\begin{array}{l}\text { Temperature }>38^{\circ} \mathrm{C} \text { at the time } \\
\text { of diagnosis }\end{array}$ & 0.29 & $0.12-0.73$ & .008 \\
$\begin{array}{l}\text { Adequacy of the overall } \\
\text { antimicrobial treatment }\end{array}$ & 0.34 & $0.14-0.84$ & .02 \\
$\begin{array}{l}\text { AOR, Adjusted odds ratio; } C I \text {, confidence interval. } \\
\end{array}$ &
\end{tabular}


mortality rate of patients with IE, varying between $9.6 \%$ and $26 \% .{ }^{18}$ The outcome is influenced by patient characteristics (ie, age, comorbidities, prosthetic valve, and diabetes mellitus), the occurrence of cardiac and noncardiac complications (ie, heart or renal failure, septic shock, stroke), the causative organism ( $S$ aureus, gram-negative bacilli, fungus), and echocardiographic findings (ie, periannular complications, large vegetations, pulmonary hypertension, severe left-sided valve regurgitation). Numerous studies have examined the outcome of patients' need for surgery during the active phase of IE. In-hospital mortality rate varies from $5.7 \%$ to $36 \% .^{2-10}$ Although identified prognostic factors differ from 1 study to another, it appears that outcome could be improved by early surgery and is impaired by the presence of the following factors: low left ventricular ejection fraction, severe congestive heart failure, pulmonary edema, preoperative shock, septic shock, prosthetic valve endocarditis, paravalvular abscess, infection owing to Staphylococcus spp or S aureus, large $(>15 \mathrm{~mm})$ vegetations, emergency surgery, persistent infection, and renal failure. , $^{3,6-11}$

To the best of our knowledge, the role of antimicrobial therapy and the impact of its adequacy on prognosis of IE have never been clearly studied, and no study has specifically focused on the prognostic impact of antimicrobial treatment associated with surgery during the active phase of IE. As demonstrated by Kollef, ${ }^{12}$ Ibrahim, ${ }^{13}$ and their coworkers more than 10 years ago, an inadequate antimicrobial therapy impaired the prognosis of critically ill patients and of those with bloodstream infections. In a previous study, including 108 patients requiring surgery during active IE for which the adequacy of antimicrobial treatment was evaluable, $\mathrm{we}^{19}$ found a 3-fold higher mortality rate $(25.8 \%$ vs $9 \%)$ when antimicrobial treatment was inadequate. In the present work, we found similar results inasmuch as operative mortality was $9.8 \%$ when antimicrobial treatment was adequate and $27.5 \%$ when it was not. Moreover, the adequacy of antimicrobial treatment associated with surgery appears as an independent prognostic factor in multivariate analysis. This result highlights the role of the infectious disease specialist collaboration with cardiologists, cardiac surgeons, and anesthesiologists as a multidisciplinary team.

The results of our prognostic analysis require further comments. As previously reported, the causative organism could influence prognosis, and it is currently admitted that staphylococcal IE has a worse outcome than IE owing to other organisms. However, in our series, mortality rates are similar in staphylococcal IE $(15.8 \%)$ and in IE owing to other organisms $(14.7 \%)$. Similarly, large $(>15 \mathrm{~mm})$ vegetations have no prognostic impact in our series. Prosthetic IE is associated in our series, as in numerous others, with a higher mortality rate $(30.5 \%$ vs $12.7 \%)$. Nevertheless, it does not appear as a significant independent prognostic factor, probably because of a too-low sample size $(n=23)$. There is no clear explanation about other discrepancies between our results and those reported in the literature. We could simply remark that results of previous studies ${ }^{2-10}$ are heterogeneous and that such differences are often observed. The last point discussed is about the prognostic impact of temperature greater than $38^{\circ} \mathrm{C}$ at the time of diagnosis that appears as an independent factor improving the outcome. This criterion is 1 of the minor modified Duke criteria for the diagnosis of IE. It was systematically collected in our database and, so, was included in our prognostic analysis. Such a result could appear surprising and be considered as a false result owing to a bias of the statistical analysis. However, recent data about association between fever and outcome of severely ill patients with infection suggest that our result could be plausible. Young and coworkers ${ }^{20}$ demonstrated that an elevated peak temperature in the first 24 hours in the ICU was associated with decreased in-hospital mortality. Although there is no definite explanation to their results, the authors remembered that fever causes direct inhibition of a number of bacteria and increases in vitro antibiotic activity.

This study had several limitations. First, our study is based on a retrospective monocentric analysis of the medical charts of all consecutive patients admitted to the Cardiovascular Surgery Department of Hôpital Cardiologique de Lille for surgical management of suspected IE. Data were retrospectively collected by an independent group on a standardized report. These points associated with stringent inclusion criteria led to exclusion from the cohort of 371 patients who underwent surgery during the study period, 140 patients for whom the diagnosis of definite or possible and active IE was not retained, and 58 patients for whom the antimicrobial treatment was not evaluable. Similarly, parameters such as evaluation of left ventricular function, exact timing of the surgical procedure, and precise echocardiographic findings were sometimes lacking, leading to exclusion of some of them from the prognostic analysis. Third, the definition of the adequacy of antimicrobial treatment could appear insufficiently precise since based only on the duration of treatment and the antibiotic(s) used comparatively to current guidelines. However, in most studies, an inadequate treatment is only defined as microbiologic documentation of an infection that is not being effectively treated. ${ }^{21}$ Finally, there is no long-term follow-up. However, inasmuch as most studies on the prognostic impact of antimicrobial treatment demonstrated a relationship between adequacy and hospital mortality, ${ }^{12,13}$ we chose to focus on operative mortality.

In conclusion, the in-hospital outcome of patients undergoing valve surgery during active IE was impaired when infection occurred in patients aged more than 60 years. Conversely, temperature greater than $38^{\circ} \mathrm{C}$ at the time of diagnosis and adequacy of the overall antimicrobial treatment have improved the prognosis. 


\section{References}

1. Murdoch DR, Corey GR, Hoen B, Miró JM, Fowler VG Jr, Bayer AS, et al International Collaboration on Endocarditis-Prospective Cohort Study (ICEPCS) Investigators. Clinical presentation, etiology, and outcome of infective endocarditis in the 21st century: the International Collaboration on Endocarditis-Prospective Cohort Study. Arch Intern Med. 2009;169:463-73.

2. Jault F, Gandjbakhch I, Rama A, Nectoux M, Bors V, Vaissier E, et al. Active native valve endocarditis: determinants of operative death and late mortality. Ann Thorac Surg. 1997;63:1737-41.

3. Alexiou C, Langley SM, Stafford H, Lowes JA, Livesey SA, Monro JL. Surgery for active culture-positive endocarditis: determinants of early and late outcome. Ann Thorac Surg. 2000;69:1448-54.

4. Thuny F, Beurtheret S, Gariboldi V, Mancini J, Avierinos JF, Riberi A, et al. Outcome after surgical treatment performed within the first week of antimicrobial therapy during infective endocarditis: a prospective study. Arch Cardiovasc Dis. 2008;101:687-95.

5. Nadji G, Goissen T, Brahim A, Coviaux F, Lorgeron N, Tribouilloy C. Impact of early surgery on 6-month outcome in acute infective endocarditis. Int J Cardiol. 2008;129:227-32.

6. Revilla A, López J, Vilacosta I, Villacorta E, Rollán MJ, Echevarría JR, et al. Clinical and prognostic profile of patients with infective endocarditis who need urgent surgery. Eur Heart J. 2007;28:65-71.

7. Musci M, Siniawski H, Pasic M, Weng Y, Loforte A, Kosky S, et al. Surgical therapy in patients with active infective endocarditis: seven-year single centre experience in a subgroup of 255 patients treated with the Shelhigh stentless bioprosthesis. Eur J Cardiothorac Surg. 2008;34:410-7.

8. Rekik S, Trabelsi I, Maaloul I, Hentati M, Hammami A, Frikha I, et al. Short- and long-term outcomes of surgery for active infective endocarditis: a Tunisian experience. Interact Cardiovasc Thorac Surg. 2009;9:241-5.

9. David TE, Gavra G, Feindel CM, Regesta T, Armstrong S, Maganti MD. Surgical treatment of active infective endocarditis: a continued challenge. J Thorac Cardiovasc Surg. 2007;133:144-9.

10. Jassal DS, Neilan TG, Pradhan AD, Lynch KE, Vlahakes G, Agnihotri AK, et al. Surgical management of infective endocarditis: early predictors of short-term morbidity and mortality. Ann Thorac Surg. 2006;82:524-9.
11. Kang DH, Kim YJ, Kim SH, Sun BJ, Kim DH, Yun SC, et al. Early surgery versus conventional treatment for infective endocarditis. NEngl J Med. 2012;366:2466-73.

12. Kollef MH, Sherman G, Ward S, Fraser VJ. Inadequate antimicrobial treatment of infections: a risk factor for hospital mortality among critically ill patients. Chest. 1999;115:462-74.

13. Ibrahim EH, Sherman G, Ward S, Fraser VJ, Kollef MH. The influence of inadequate antimicrobial treatment of bloodstream infections on patient outcomes in the ICU setting. Chest. 2000;118:146-55.

14. Petrak RM, Sexton DJ, Butera ML, Tenenbaum MJ, MacGregor MC, Schmidt ME, et al. The value of an infectious diseases specialist. Clin Infect Dis. 2003;36:1013-7.

15. Esposito S, Leone S. Antimicrobial treatment for Intensive Care Unit (ICU) infections including the role of the infectious disease specialist. Int J Antimicrob Agents. 2007;29:494-500.

16. Li JS, Sexton DJ, Mick N, Nettles R, Fowler VG Jr, Ryan T, et al. Proposed modifications to the Duke criteria for the diagnosis of infective endocarditis. Clin Infect Dis. 2000;30:633-8.

17. Mouly S, Ruimy R, Launay O, Arnoult F, Brochet E, Trouillet JL, et al. The changing clinical aspects of infective endocarditis: descriptive review of 90 episodes in a French teaching hospital and risk factors for death. J Infect. 2002;45:246-56.

18. Habib G, Hoen B, Tornos P, Thuny F, Prendergast B, Vilacosta I, et al. ESC Committee for Practice Guidelines. Guidelines on the prevention, diagnosis, and treatment of infective endocarditis (new version 2009): the Task Force on the Prevention, Diagnosis, and Treatment of Infective Endocarditis of the European Society of Cardiology (ESC). Endorsed by the European Society of Clinical Microbiology and Infectious Diseases (ESCMID) and the International Society of Chemotherapy (ISC) for Infection and Cancer. Eur Heart J. 2009;30:2369-413.

19. Fayad G, Leroy G, Devos P, Hervieux E, Senneville E, Koussa M, et al. Characteristics and prognosis of patients requiring valve surgery during active infective endocarditis. J Heart Valve Dis. 2011;20:223-8.

20. Young PJ, Saxena M, Beasley R, Bellomo R, Bailey M, Pilcher D, et al. Early peak temperature and mortality in critically ill patients with or without infection. Intensive Care Med. 2012;38:437-44.

21. Kollef MH. Inadequate antimicrobial treatment: an important determinant of outcome for hospitalized patients. Clin Infect Dis. 2000;31(Suppl 4):S131-8. 